\title{
Influence of aluminium sheet surface modification on the self-piercing riveting process and the joint static lap shear strength
}

\author{
Dezhi Li ${ }^{1}$ \\ Received: 31 March 2017 / Accepted: 23 June 2017 /Published online: 7 July 2017 \\ (C) The Author(s) 2017. This article is an open access publication
}

\begin{abstract}
Self-piercing riveting (SPR) has been widely used in automotive as one of the major joining technologies for aluminium structures due to its advantages over some of the more traditional joining technologies. Research has shown that friction is a very important factor that influences both the riveting process and the joint strength for SPR, but these influences have not been fully understood. In this paper, AA5754 sheets with different surface textures, such as original with solid wax, hot water washed, sandpaper ground and grit blasted, were used to study the influence of friction on therivet inserting process, joint features and static lap shear strength. The results of joint features and rivet setting displacement-force curve showed that hot water wash and sandpaper grinding on aluminium sheet did not have significant influence on the rivet inserting process and joint features; however, for joints with grit-blasted substrates, the rivet setting forces were higher at the beginning, and a middle section of the curve and the joint features, such as interlocks and minimum remaining bottom material thickness $\left(T_{\min }\right)$, were clearly altered. The lap shear tests showed that hot water washing can slightly increase the lap shear strength, sandpaper grinding increased the static lap shear strength further and grit blasting increased the static lap shear strength the most.
\end{abstract}

Keywords Self-piercing riveting $\cdot$ Friction $\cdot$ Rivet setting process $\cdot$ Lap shear strength $\cdot$ Displacement-force curve

\section{Dezhi Li}

dezhi.li@warwick.ac.uk

1 WMG, University of Warwick, Coventry CV4 7AL, UK

\section{Introduction}

Aluminium has been proved having a great potential as to weight-reduction when it is used to replace traditional steel structures in automotive body structures to increase fuel efficiency and reduce $\mathrm{CO}_{2}$ emission. Self-piercing riveting (SPR) is one of the main joining methods for aluminium intensive automotive body structures due to its advantages in the working environment, ability to join dissimilar materials, ability to join with adhesives, low-energy requirement and high static and fatigue-joint strengths [1-4]. A lot of research results on SPR have been reported, such as on rivetability $[5,6]$, rivet materials [7, 8], rivet coatings [9], joint strength [10-13], rivet geometry [14] and joint dimensions [12, 15].

Friction is very important for both the rivet setting process and joint strength due to the relative movements between different components. The main frictions, existing in a SPR setting process or a SPR joint, are between sheet materials and between rivet and sheet materials. There are also frictions between the blank holder and the top sheet and between the bottom sheet and the die for the SPR setting process. As we know, friction between two surfaces is directly related with the surface condition. To find out the influence of surface conditions on the SPR setting process and joint strength, some research had been conducted.

Han and Chrysanthou [16] and Han et al. [17] studied the influence of coatings on sheet material on the joint quality and mechanical strength of SPR joints. In their study, AA5754 was used as the top sheet, and HSLA 350 with different coatings, i.e. uncoated, e-coated and zinc-plated, was used as the bottom sheet. The surface roughness of zinc-plated HSLA 350 was higher than that of the e-coated one but lower than that of the uncoated one. Their results showed that the extent of the effects of surface coatings on the joint quality and mechanical behaviour of SPR joints differed significantly with different 
types of coatings on the HSLA steel. Han et al. [18] also studied the influence of sheet/sheet interfacial condition on the fatigue performance of SPR joints. The results showed that the presence of a wax-based solid surface lubricant could delay the onset of fretting damage on the alloy surface, leading to extended fatigue life; the application of a PTFE insert at the interface between the riveted sheets eliminated or significantly reduced fretting damage but led to a reduction in the fatigue life due to a different failure mode. Results from Li et al. [13] showed that fretting during fatigue increased the surface roughness, which consequently increased the remaining static lap shear strength of the specimens due to the increased friction force between the tip of the punched hole in the top sheet and the edge of the partially pierced hole in the bottom sheet.

In this paper, the influence of different surface conditions of aluminium AA5754 through surface modification on rivet inserting process, joint features and static lap shear strength was studied.

\section{Experimental procedure}

\subsection{Materials}

The material used in this study is commercially available $2.0 \mathrm{~mm}$ thick AA5754 with a standard pre-treatment (PT2) and wax lubricant (AL070). The AA5754 is an Mg-rich Al alloy, and it has UTS of $241 \mathrm{MPa}$, yield strength of $110 \mathrm{MPa}$ and elongation of $25 \%$. The wax layer on the surface of AA5754 is a solid lubricant, which is very important for stamping for reducing the friction between parts and stamping press.

\subsection{Surface modification}

In order to see the influence of surface texture, such as lubricant, roughness and friction coefficient, on the SPR riveting process and joint strength, certain surfaces around the joining area of the sheet materials before rivet inserting were modified with different methods, i.e. washing with hot tap water (around $60{ }^{\circ} \mathrm{C}$ ), grinding with P120 sandpaper and grit blasting. After blasting, the coupons were blown with a compress air gun to remove the residual grit particles as much as possible. The target surfaces are the bottom surface of the top sheet and/or the top surface of the bottom sheet. However, when washed with hot tap water, the other side of the sheet material would be affected as well, since it was also exposed to the hot water. For joint quality analysis and SPR setting process study, $38 \mathrm{~mm}$ square coupons were used; for lap shear tests, $111.5 \mathrm{~mm} \times 48 \mathrm{~mm}$ coupons were used. For the $38 \mathrm{~mm} \times 38 \mathrm{~mm}$ coupons, the modified area is the whole coupon area (either the bottom surface of the top sheet or both the bottom surface of the top sheet and the top surface of the bottom sheet); for the coupons for lap shear specimens, the modified areas are about $23-30 \mathrm{~mm} \times 48 \mathrm{~mm}$ at the overlapping end on both the bottom surface of the top sheet and the top surface of the bottom sheet.

\subsection{Sample preparation}

The basic stack used for this study is a 2-mm AA5754 top sheet joined to a 2-mm AA5754 bottom sheet, and thereafter called (2+2)AA5754. For all joints, $6.5 \mathrm{~mm}$ long steel rivets with a countersunk head and mechanical zinc/tin surface coating were used. The rivets were supplied by Henrob Ltd., and all samples were produced using the Henrob servo-driven SPR system with a fixture to ensure an accurate and consistent riveting location. A rivet/die/velocity combination, as listed in Table 1, was selected to achieve good joint features/quality. The displacement-force curves for rivet inserting processes were recorded with an Emhart Tucker SPR system with the same rivet/die combination and an equivalent setting force to achieve equivalent joint features. Joint quality of specimens was inspected through cross sections. A special fixture was used to ensure all joints were vertically cross-sectioned through the centre of the rivets. Following sectioning, the joint features were measured and analysed with respect to rivet head height, interlock and minimum remaining bottom material thickness using the a4i image analysis software supplied by Aquinto. At least three joint cross-sections were measured for each joint combination.

Specimen geometries and dimensions for lap shear tests are shown in Fig. 1. During the preparation of lap shear specimens, coupons were cut from the sheet such that the longitudinal direction of coupons (loading direction during subsequent mechanical tests) coincides with the rolling direction of sheet metal. To reduce any variations of rivet position, a custom-designed fixture was used to set rivets into correct positions. For each specimen, the coupon width was fixed at $48 \mathrm{~mm}$, and two rivets were set with an edge distance of $11.5 \mathrm{~mm}$, which was recommended by Li et al. [12, 15] for optimum static and fatigue SPR joint strength.

\subsection{Mechanical tests}

Mechanical tests were conducted by following the company standards. A bench-top Instron with a cross-head speed of $10 \mathrm{~mm} / \mathrm{min}$ was used for the static tests, and in order to minimize coupon-bending during lap shear testing, $2 \mathrm{~mm}$ thick spacers were applied at the two clamping ends of the lap shear

\begin{tabular}{ll} 
Table 1 & Optimum SPR parameters for (2+2)AA5754 stack-up \\
\hline Rivet & Length, $6.5 \mathrm{~mm}$; type, countersunk; hardness, $410 \mathrm{Hv}$ \\
Die & Cavity diameter, $9 \mathrm{~mm}$; cavity depth, $2 \mathrm{~mm}$; type, flat bottom \\
Velocity & 100 (Henrob unit, determining applied force) \\
\hline
\end{tabular}




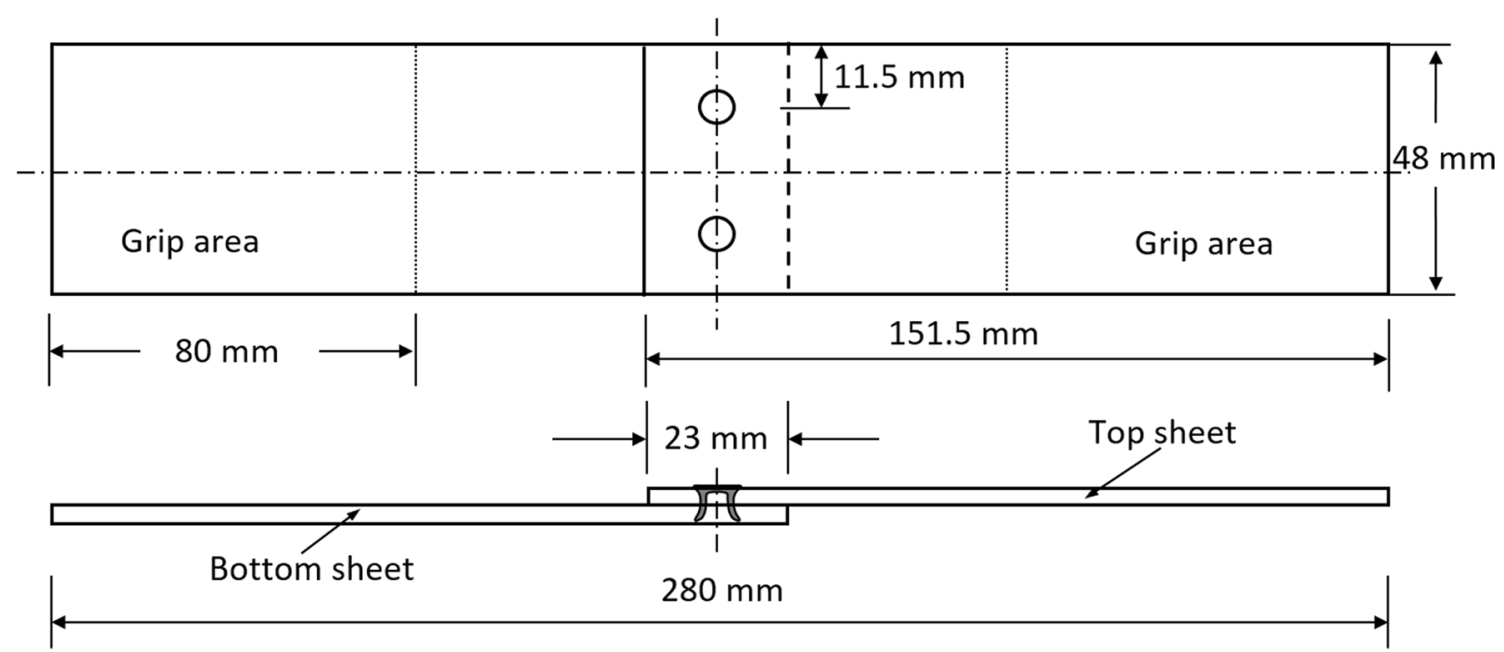

Fig. 1 Specimen geometry for lap shear tests

samples. At least three specimens were tested for each joint combination.

\subsection{Friction tests}

The friction behaviour between two fresh AA5754 friction pads and the specific surfaces of a 2 mm thick AA5754 test strip was measured using a custom-made device, as shown in Fig. 2, attached to an Instron tensile test machine. The bottom of this device was mounted on the platform of the Instron; the test strip was fixed on the grip of the Instron with the strip passing between the fixed and the moving pads (with smaller friction pads attached) of the device. During a friction test, a pressure was applied in the pneumatic system, and a force would be transferred to the piston through the lever and subsequently to the friction test pads. After each test, the worn friction pads would be removed, and a pair of fresh pads would be attached for the next test. The dimension of the friction pads was $14 \mathrm{~mm} \times 14 \mathrm{~mm}$ with $\sim 1 \mathrm{~mm}$ chamfer at the four edges on the contact surface (contact area was about $12 \mathrm{~mm} \times 12 \mathrm{~mm}$ ); the dimension of the test strips was $25 \mathrm{~mm} \times 330 \mathrm{~mm}$. Two contact pressures were used, and they were 4.58 and $22.92 \mathrm{MPa}$, corresponding to the compressed air pressure of 1 and 5 bar, respectively. The 1 bar pressure is the minimum accurate pressure that can be controlled by the compressed air regulator, which was used to produce a lowload friction; the 5 bar pressure was used to produce a highload friction as comparison.
Fig. 2 Friction-test device (the actual friction pads are attached in the middle of the moving pad and the fixed pad)

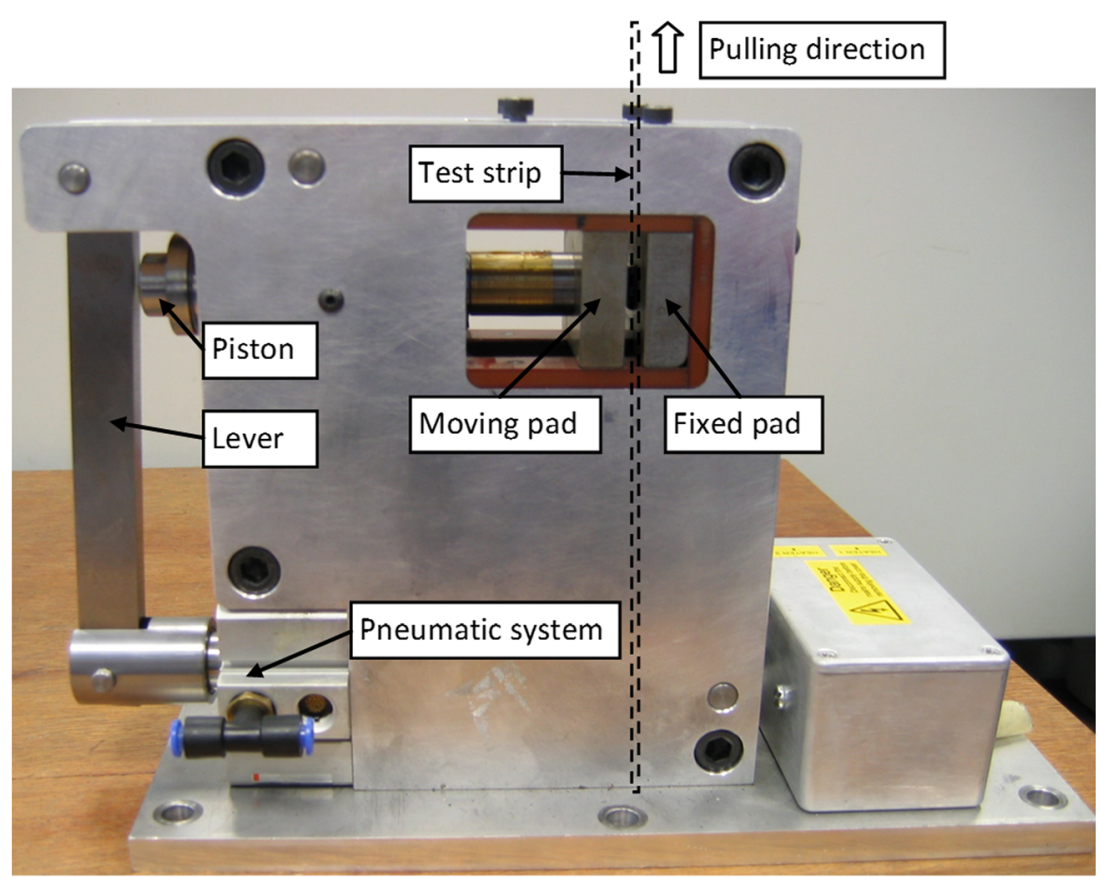


Fig. 3 Cross section of a reference $(2+2)$ AA5754 SPR joint with original surface texture and lubricant

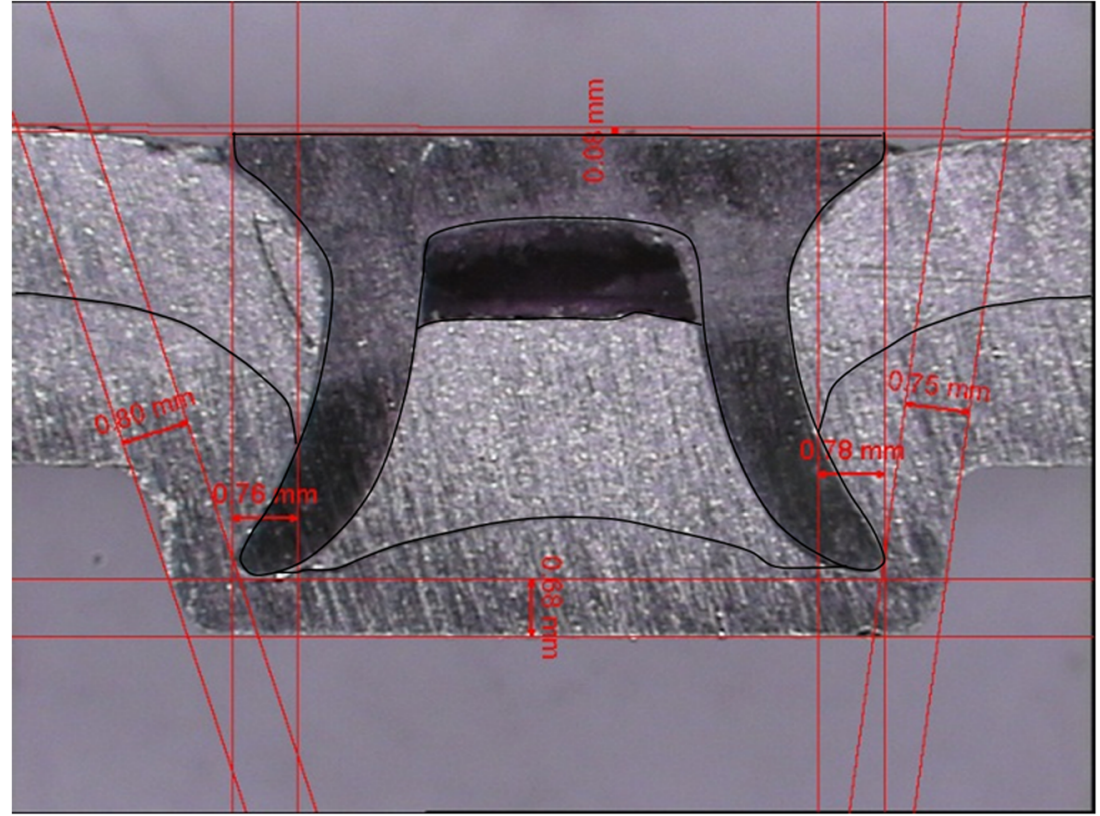

measured and annotated, and these are rivet head height of $-0.08 \mathrm{~mm}$, an average interlock of $0.77 \mathrm{~mm}$ and a minimum remaining bottom material thickness of $0.68 \mathrm{~mm}$.

Figure 4 shows the cross sections of (2+2)AA5754 SPR joints with certain surfaces modified by washing with hot tap water and grinding with P120 sandpaper. Since the virgin AA5754 sheet material has a wax lubricant (AL070) on the surface, washing with hot water (around $60{ }^{\circ} \mathrm{C}$ ) will melt and wash a large amount of wax away although not completely remove it; grinding with sandpapers will also remove some of the wax away and make the surface rougher. As a result, the frictions at the top and bottom sheets interface would be increased after washing with hot water and grinding with sandpapers. From Figs. 3 and 4, it can be seen that for the joints with surfaces modified by washing with hot water or grinding with the P120 sandpaper, the interlock distances did not have obvious change compared to the reference joint, but the rivet head height and the minimum remaining bottom material

Figure 3 shows a cross section of the reference SPR joint studied in this paper. The SPR joint quality attributes were

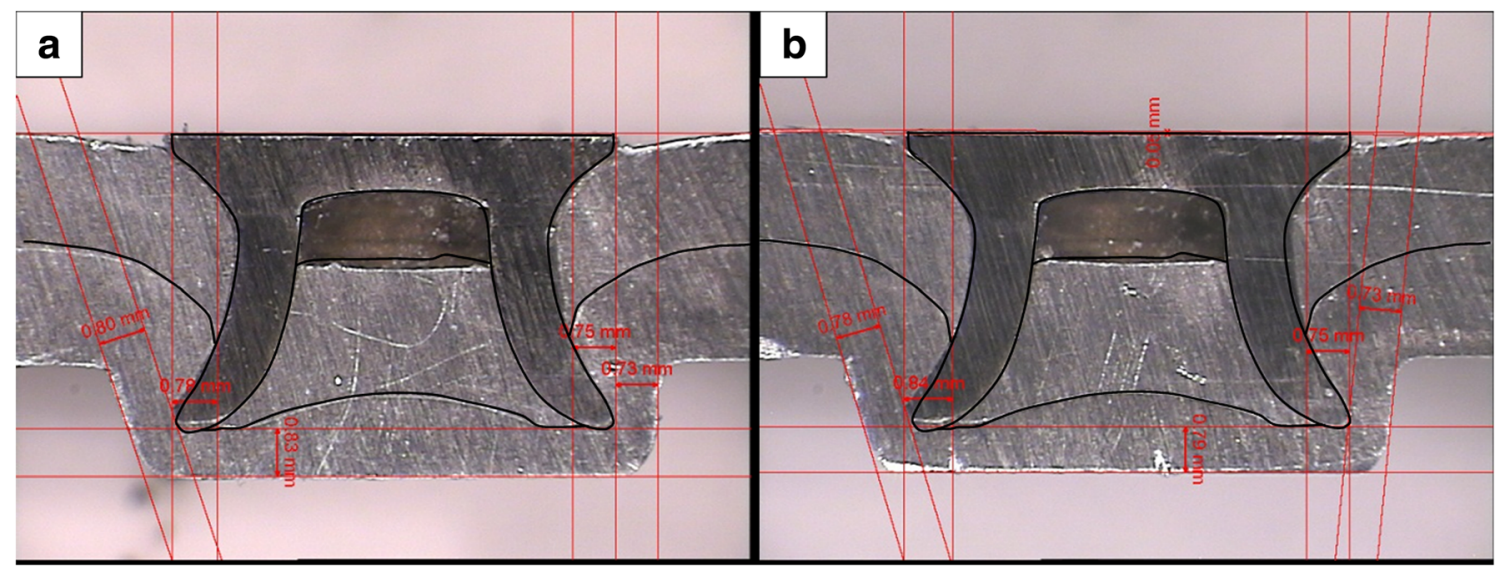

Fig. 4 Cross section of the (2+2)AA5754 SPR joints with the bottom surface of the top sheet modified by a washing with hot tap water and $\mathbf{b}$ grinding with P120 sandpaper (the black outline is the profile of the deformed rivet and sheet materials in the reference joint) 


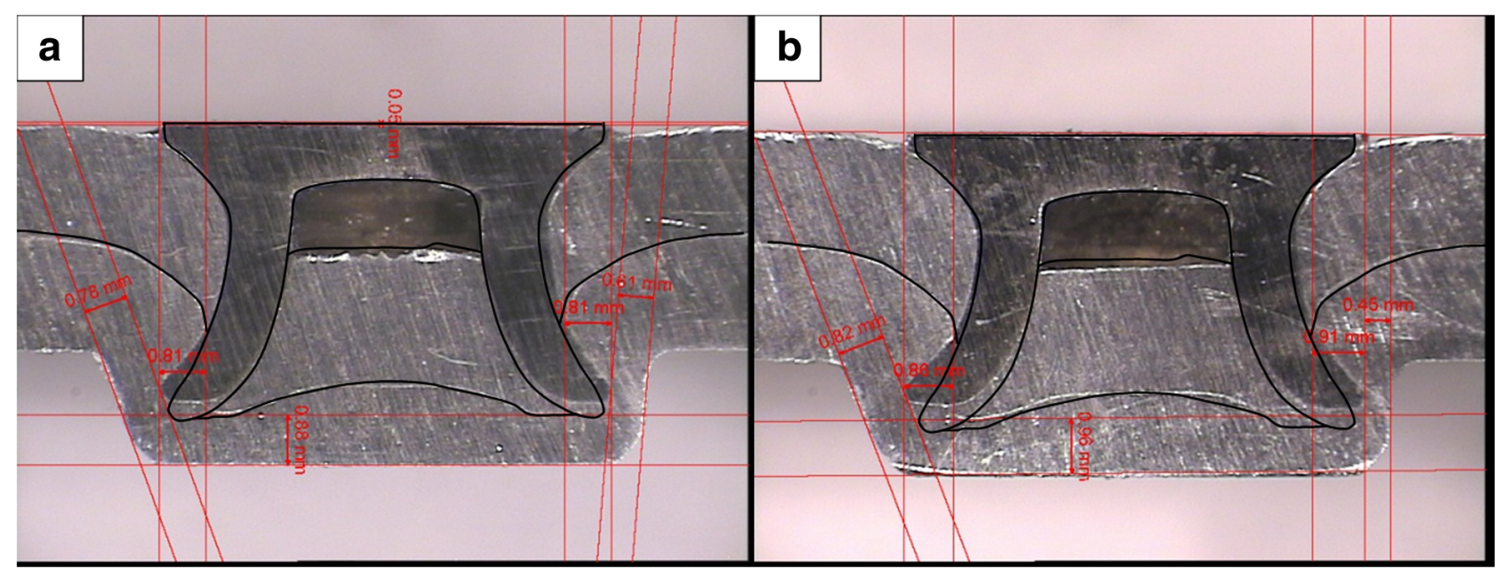

Fig. 5 Cross section of the (2+2)AA5754 SPR joints with certain surface modified by a grit blasting on the bottom surface of the top sheet and $\mathbf{b}$ grit blasting on the bottom surface of the top sheet and the top surface of the bottom sheet

thickness slightly increased. By comparing the outlines of the deformed rivets and sheet materials with those from the reference joint, no significant difference can be seen.

Figure 5 shows the cross sections of (2+2)AA5754 SPR joints with certain surfaces modified by blasting. It can be seen that for the joints with surfaces modified by grit blasting, the interlock distances had an obvious increase; the rivet head height increased slightly; however, the minimum remaining bottom material thickness was reduced greatly. This reduction of the minimum remaining bottom material thickness was caused by the material thinning between the rivet tail and the side edge of the joint button, although the minimum remaining bottom material thickness to the bottom surface of the joint button was actually increased. By comparing the outlines of the deformed rivets with those from the reference joint, it can be seen that the rivets in these two types of joints had much larger bending deformation than that in the reference joint.

Table 2 summarizes the joint features of some SPR joints. It can be seen that when the sheet materials were modified by washing with hot water and grinding with sandpapers, the rivet head height and the minimum remaining bottom material thickness to the bottom surface of the joint button slightly increased, but the average interlocks for the joints did not have an obvious increase. When the sheet materials were modified by grit blasting, the rivet head height and the interlock distance had an obvious increase and the overall $T_{\min }$ and the $T_{\min }$ to the sides of joint buttons became much thinner, although the $T_{\min }$ to the bottom of joint buttons increased.

\subsection{Surface texture and roughness}

Figure 6 shows the surface textures of 2 mm AA5754 with different surface modifications. From Fig. 6b, it can be seen that after washing with hot tap water, the surface texture of AA5754 did not have an obvious change, but the rolling tracks can be seen more clearly because some of the solid wax lubricant was washed away by the hot water. The coupon for Fig. $6 \mathrm{c}$ was ground vertical to the rolling direction. It can be seen that after grinding, the rolling tracks could not be seen any more and the surface became rougher. The surface of the coupon after grit blasting became much less reflective and more even without any track, and there were likely some grit fragments that remained on the surface according to the research from Maruyama et al. [19].

Table 3 shows the overall surface roughness (measured from two perpendicular directions) of $2 \mathrm{~mm}$ AA5754 with different surface modifications. It can be seen that hot tap water washing increased the surface roughness slightly, grinding with P120 sandpaper doubled the surface roughness and grit blasting increased the surface roughness the most. The
Table 2 The joint features of all SPR joints

\begin{tabular}{lclcrl}
\hline Joint $^{\mathrm{a}}$ & $\begin{array}{l}\text { Rivet head } \\
\text { height }(\mathrm{mm})\end{array}$ & $\begin{array}{l}\text { Average } \\
\text { interlocks }(\mathrm{mm})\end{array}$ & \multicolumn{2}{l}{$T_{\min }(\mathrm{mm})$} & \\
\cline { 5 - 6 } & & & To the bottom & To the sides & \multicolumn{1}{c}{ Overall } \\
\hline Reference & $-0.08 \pm 0$ & $0.78 \pm 0.03$ & $0.67 \pm 0.02$ & $0.7 \pm 0.07$ & $0.67 \pm 0.02$ \\
Washed + virgin & $-0.02 \pm 0.02$ & $0.77 \pm 0.03$ & $0.83 \pm 0.01$ & $0.74 \pm 0.01$ & $0.74 \pm 0.01$ \\
Washed + washed & $-0.01 \pm 0.01$ & $0.79 \pm 0.01$ & $0.85 \pm 0.02$ & $0.73 \pm 0.01$ & $0.73 \pm 0.01$ \\
P120 + virgin & $-0.03 \pm 0.04$ & $0.79 \pm 0.04$ & $0.79 \pm 0$ & $0.74 \pm 0.01$ & $0.74 \pm 0.01$ \\
Blasted + virgin & $0.06 \pm 0.01$ & $0.85 \pm 0.1$ & $0.87 \pm 0.01$ & $0.55 \pm 0.09$ & $0.55 \pm 0.09$ \\
Blasted + blasted & $0 \pm 0$ & $0.94 \pm 0.08$ & $1 \pm 0.05$ & $0.39 \pm 0.09$ & $0.39 \pm 0.09$ \\
\hline
\end{tabular}

a Modification on the top sheet + modification on the bottom sheet; 'virgin' means the original surface condition 
Fig. 6 Surface textures of $2 \mathrm{~mm}$ AA5754 with different surface modifications. a Reference. $\mathbf{b}$ Washed with hot tap water. c Ground with P120 sandpaper (grinding direction vertical to rolling). d Grit-blasted

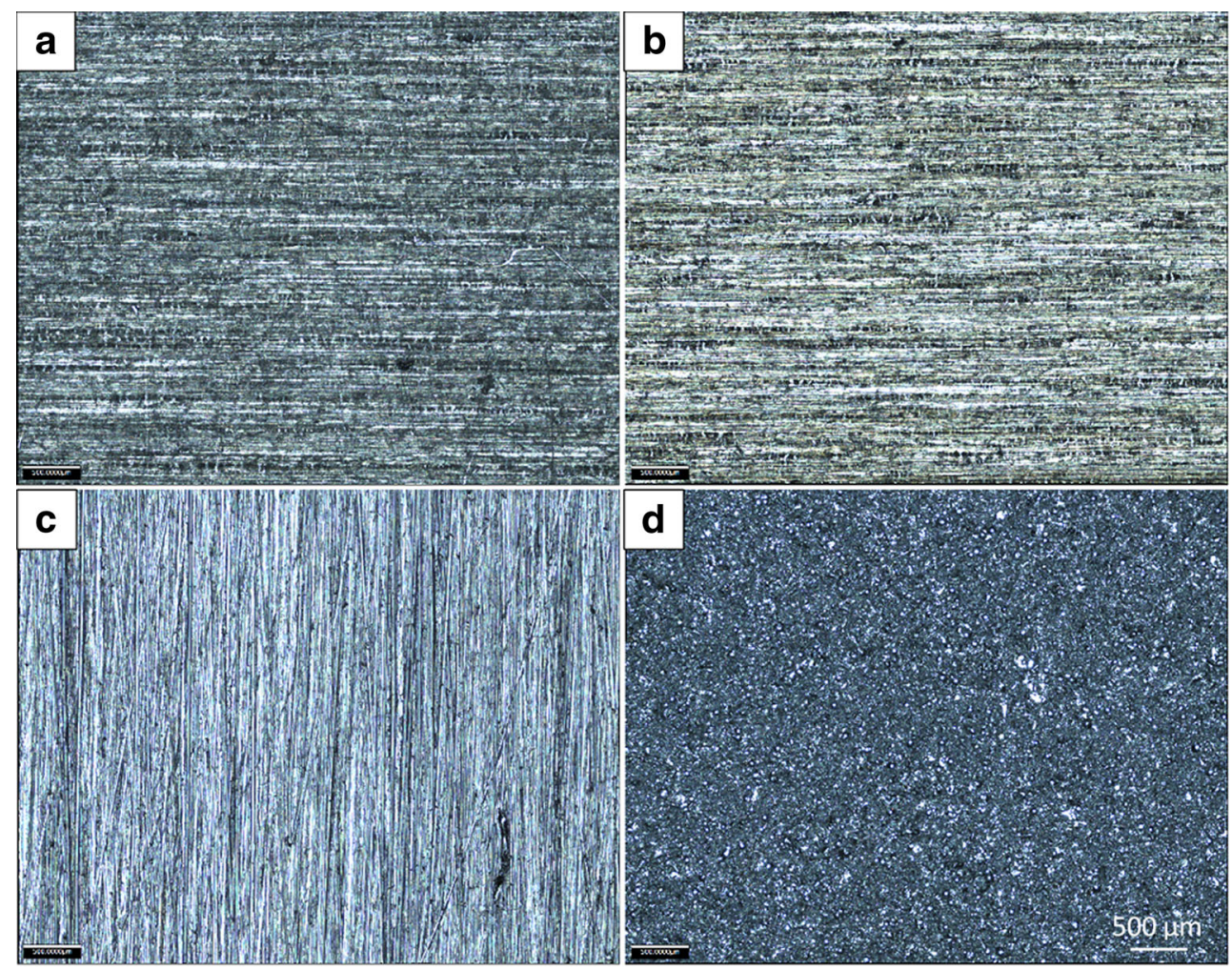

slightly increased surface roughness by hot water washing should be related to the partially removed solid lubricant on the surface. For the reference coupon and the coupon washed by the hot tap water, the roughness along the direction of vertical to the rolling direction was higher than that along the rolling direction. For the coupon ground by P120 sandpaper, the roughness along the direction of vertical to the grinding direction was higher than that along the grinding direction. For the coupon that was grit blasted, the roughness along the two directions was similar.

\subsection{Friction forces}

Table 4 shows the friction forces between the virgin AA5754 and the surface-modified AA5754 sheet material. Two gauge pressures, 1 and 5 bars, were used; the equivalent contact forces between the friction pads and the test strip were 660

Table 3 Overall surface roughness of $2 \mathrm{~mm}$ AA5754 with different surface modifications

\begin{tabular}{lrlrr}
\hline $\begin{array}{l}\text { Coupons } \\
(\mu \mathrm{m})\end{array}$ & Reference & \multicolumn{1}{l}{$\begin{array}{l}\text { Hot water } \\
\text { washed }\end{array}$} & P120 ground & Grit blasted \\
\hline$R_{\mathrm{a}}$ & $0.69 \pm 0.04$ & $0.73 \pm 0.02$ & $1.49 \pm 0.44$ & $3.08 \pm 0.07$ \\
$R_{\mathrm{q}}$ & $0.87 \pm 0.05$ & $0.95 \pm 0.03$ & $1.87 \pm 0.54$ & $3.88 \pm 0.14$ \\
$R_{\mathrm{t}}$ & $5.42 \pm 0.53$ & $6.63 \pm 0.86$ & $11.59 \pm 3.74$ & $24.15 \pm 3.53$
\end{tabular}

$R_{a}$ average roughness, $R_{q}$ root-mean-square roughness, $R_{t}$ maximum peak-to-valley height roughness and $3300 \mathrm{~N}$, respectively. The measured friction forces were double of the frictions at one pad and the strip interface, since the contacts were from the two sides of the strip.

During most of the friction tests, the friction was not stabilized after static friction due to partial ploughing of friction pads into the test strips, so the lowest friction forces after static friction could only give indication of the dynamic friction forces, and they quickly developed into much higher wearing forces. Although the repeatability of the friction tests was not good, the results did give an indication of the degree of the friction of various interfaces.

It is believed that the friction between sheet-material interface is determined by the sheet material surface conditions, such as roughness, amount of remaining lubricant and residual

Table 4 Friction forces between the virgin AA5754 (friction pads) and the surface-modified AA5754 sheets (test strips)

\begin{tabular}{cllll}
\hline $\begin{array}{l}\text { Surface } \\
\text { conditions }\end{array}$ & $\begin{array}{l}\text { Gauge } \\
\text { pressure (bar) }\end{array}$ & $\begin{array}{l}\text { Static } \\
\text { friction (N) }\end{array}$ & $\begin{array}{l}\text { Lowest force after } \\
\text { static friction (N) }\end{array}$ & $\begin{array}{l}\text { Wearing } \\
\text { force (N) }\end{array}$ \\
\hline Virgin (ref.) & 1 & 167 & 157 & 210 \\
& 5 & 300 & 215 & 450 \\
Hot water & 1 & 196 & 173 & 280 \\
washed & 5 & - & - & - \\
P120 ground & 1 & 322 & 145 & - \\
& 5 & 2850 & 1450 & 3900 \\
Grit blasted & 1 & 470 & 375 & 1400 \\
& 5 & 3625 & 2668 & 6000 \\
\hline
\end{tabular}



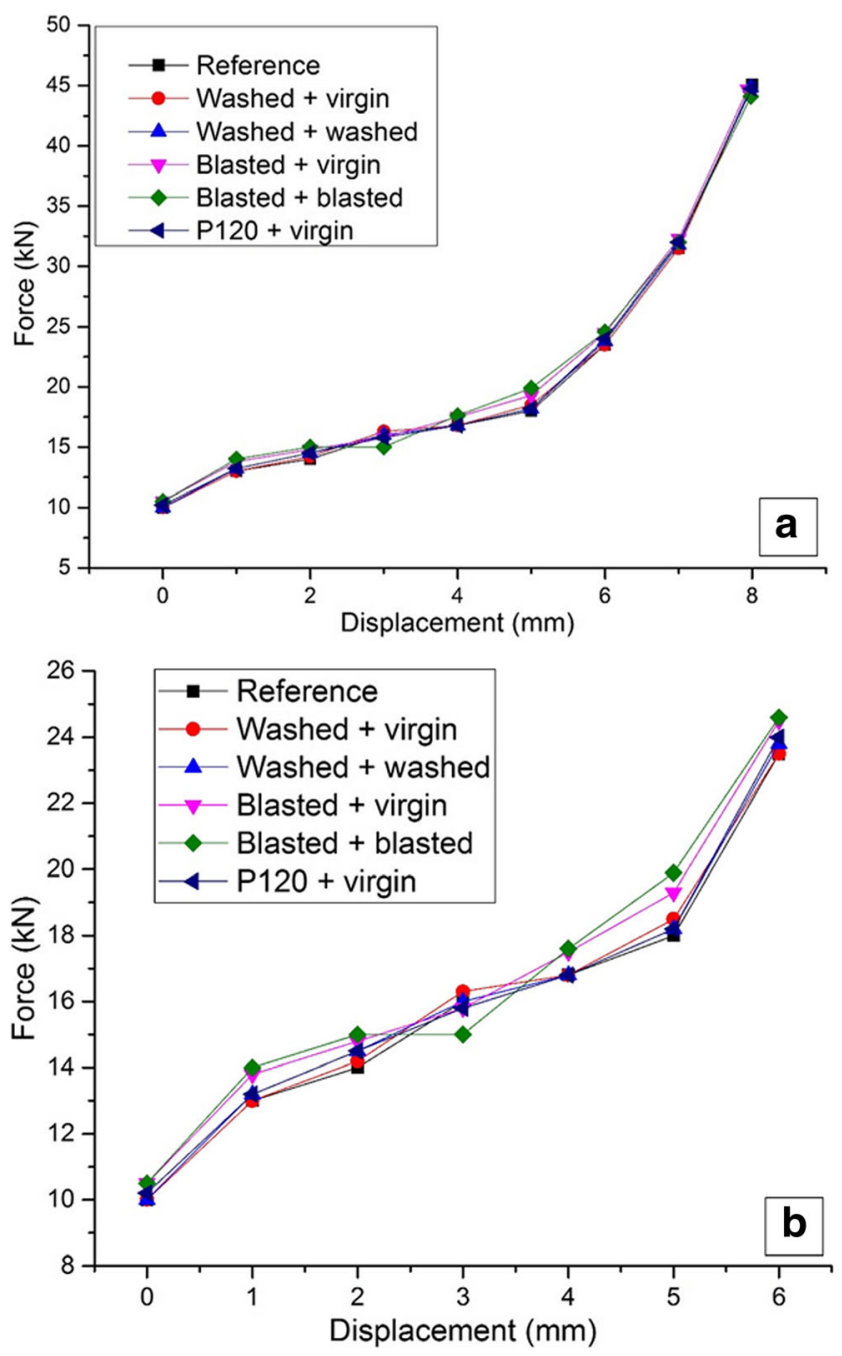

Fig. 7 SPR rivet setting displacement-force curves (b is the local enlarge of $\mathbf{a}$ ) hard particles (from grit blasting). Increased surface roughness, reduced lubricant and embedded hard particles will increase interface friction. From Table 3, it can be seen that by washing with hot tap water, grinding with P120 sandpaper and grit blasting, the surface roughness of AA5754 was increased. These surface modifications also reduced the amount of solid lubricant on the surface. From Table 4, it can be seen that washing the strips with hot water could slightly increase the friction forces; grinding with P120 sandpaper could further increase the friction forces; grit blasting increased the friction forces the most. Comparing Tables 3 and 4, it can be seen that the measured static friction forces at low contact force were roughly proportional to the surface roughness. Table 4 also shows that with a larger contact force (gauge pressure) the increase of friction forces due to surface modification would be much larger.

\subsection{SPR rivet inserting process}

Figure 7 shows the SPR rivet setting displacement-force curves for joints with and without local area surface modification. It can be seen that washing with hot tap water and grinding with P120 sandpaper did not have obvious influence on the rivet setting displacement-force curves. For the sheet materials modified by grit blasting, the rivet setting forces were slightly higher between displacements of 0 and 2 and displacements of 4 and 7, but there was no obvious influence at the end of the rivet setting process; around displacement of 3 , the rivet setting forces were similar or even lower than that for the original sheet materials (reference).
Fig. 8 Quasi-static lap shear strength of the specimens

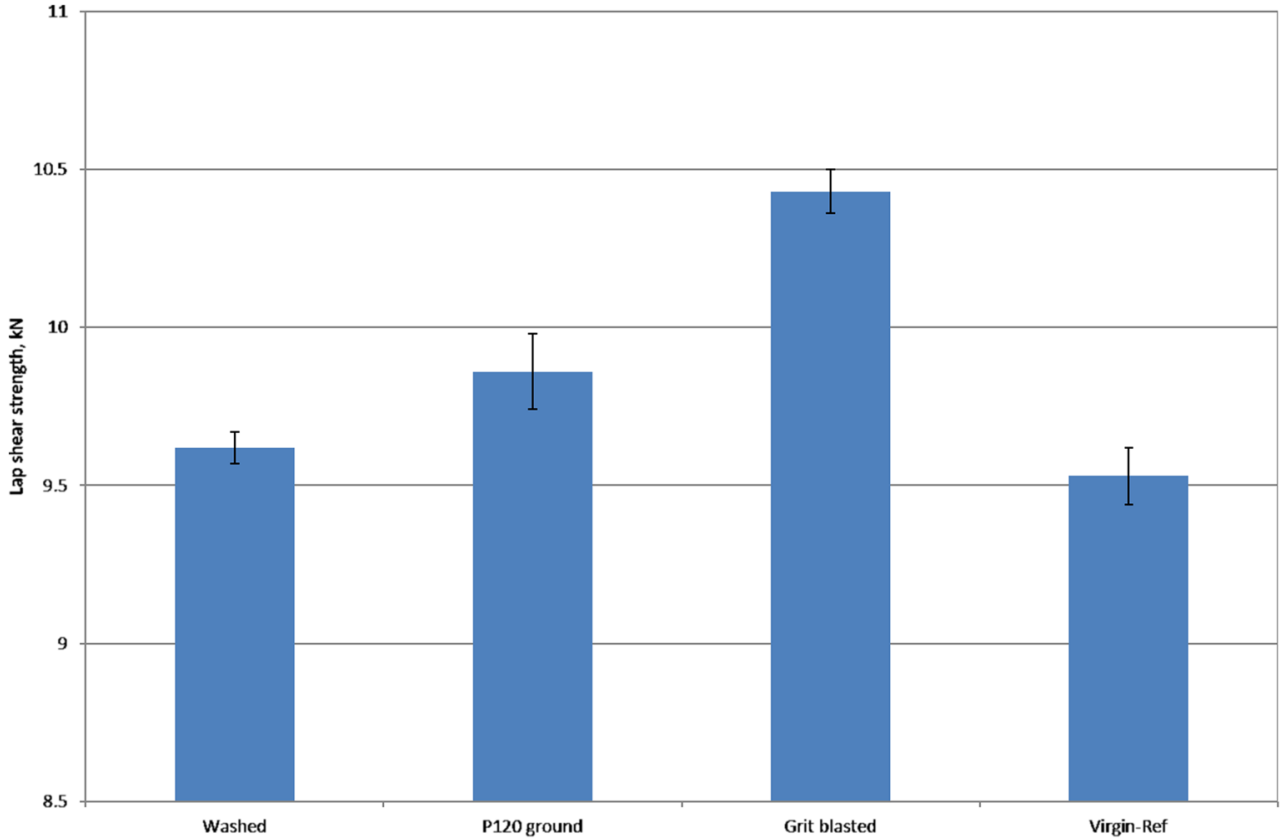




\subsection{SPR joint static lap shear strength}

Figure 8 presents the quasi-static lap shear strength of various specimens with or without surface modification. The surface modification was conducted on the top surface of the bottom sheet and the bottom surface of the top sheet. It can be seen that washing the sheet materials with hot tap water slightly increased the joint lap shear strength; grinding the sheet materials with rough sandpapers (P120) could further increase the joint lap shear strength; and grit blasting the sheet materials increased the joint lap shear strength the most.

\section{Discussion}

\subsection{Influence of sheet surface textures/friction on SPR setting process and joint quality}

From the results in Sects. 3.1 and 3.4, it can be seen that different surface textures through different surface modifications had different influences on the joint quality and rivet inserting displacement-force curves. When the surface of the
AA5754 sheet material was modified through washing with hot water and grinding with P120 sandpapers, the different surface textures did not have obvious influence on the joint quality and rivet inserting process. However, when the surface of the AA5754 sheet material was modified through blasting, the joints had different joint quality due to different rivet and sheet material deformation, and the slightly higher forces were required at the beginning and in a middle section of the rivet inserting process.

It is believed that the difference on the joint quality and the rivet setting forces between the reference stack and the stack with blasted surfaces is caused by the friction difference at the top/bottom sheet interfaces. Figures 9 and 10 show the SPR rivet setting displacement-force curves with joint cross sections for the $(2+2)$ AA5754 stacks with the original surfaces and the blasted surfaces, respectively. From the joint cross sections, it can be seen that the difference of the deformation of the rivets between the two stacks is not obvious before the rivet inserting into the bottom sheet. However, after the rivet started to pierce the bottom sheet, this difference became obvious. The main frictions during a SPR rivet setting process are the friction between the rivet and the sheet materials and
Fig. 9 SPR rivet setting displacement-force curve for the $(2+2)$ AA5754 reference stack with original lubricated surface

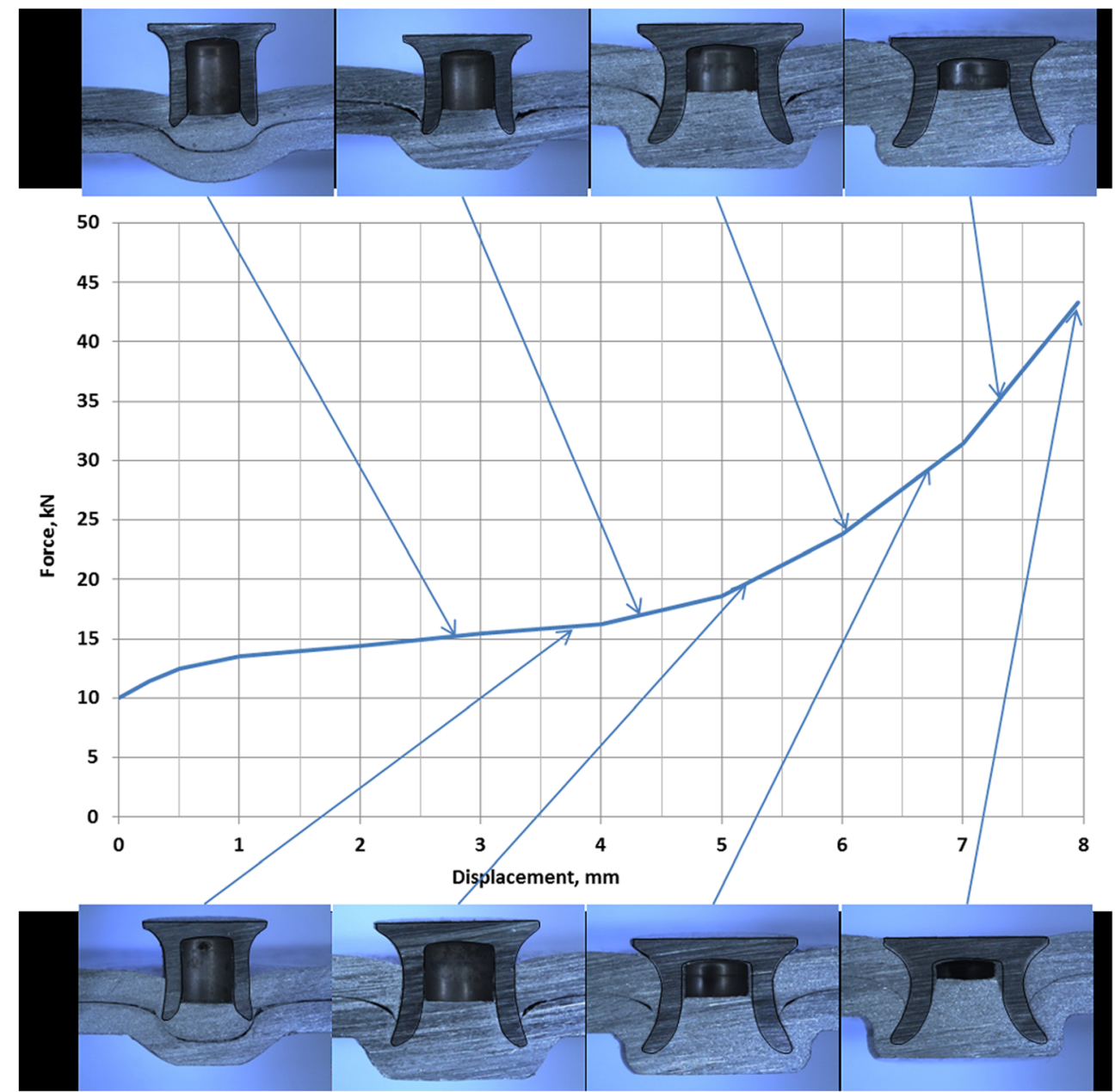


Fig. 10 SPR rivet setting displacement-force curve for the $(2+2)$ AA5754 stack with gritblasted surface (the bottom surface of the top sheet and the top surface of the bottom sheet) (the overlapped rivet contours are the contours of the rivet for the $(2+2)$ AA5754 reference stack with the original surface at the same rivet inserting stage)
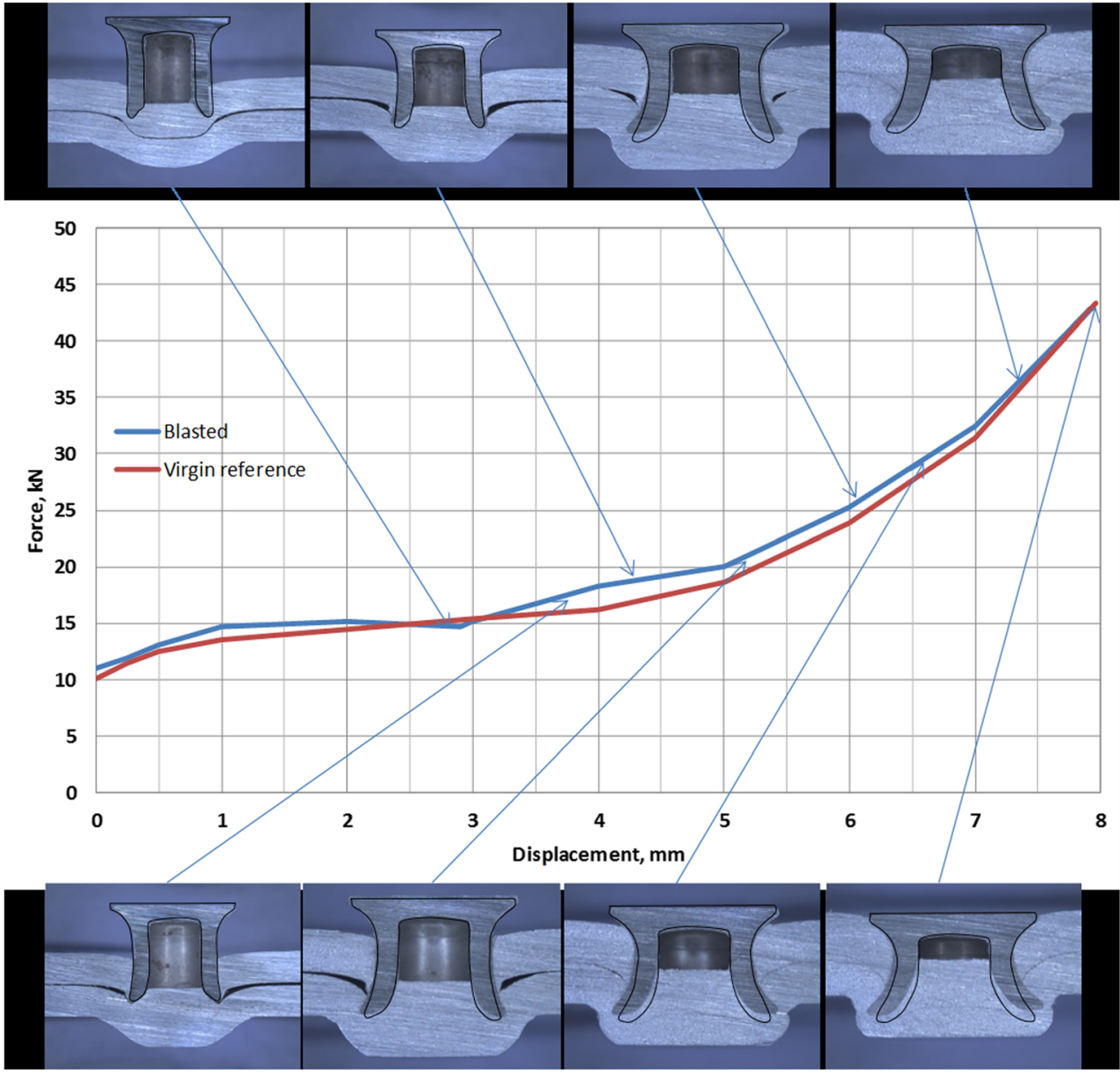

the friction between the top and the bottom materials. The friction between the rivet and the sheet material is mainly controlled by the rivet surface conditions and the bulk properties of the sheet materials. In this study, because the rivets and the bulk properties of the sheet materials were the same, the main friction that caused the difference of the rivet setting process was from the top and bottom material interfaces. It is believed that the friction between the top and the bottom sheets outside the rivet piercing area had no significant influence on the rivet setting process, because during the early stages, there was a gap between the top and the bottom sheet at the friction-sensitive area (next to the rivet inserting location), and after the gap was closed up, there was no large relative movement between the top and the bottom sheets at this location. The main influence is believed to be from friction at the interface between the punched portion of the top sheet and the bottom sheet. At the beginning stages of rivet inserting, the rivet punched through the top sheet, and the bottom material was bent into the die cavity; the bottom sheet had much larger deformation along the sheet material plane direction than the top sheet. When a large friction exists between the punched portion of the top sheet and the bottom sheet, the deformation of the bottom sheet would become less even, and as a result, necking and stress concentration would happen and a higher force would be required to set the rivet and deform the bottom sheet. After the rivet started to penetrate the bottom sheet, there were three main activities for the rivet setting process, including (i) rivet further penetrating into the bottom sheet, (ii) pushing of the punched top material into the rivet cavity and (iii) further stretching and filling of the bottom material into the die cavity. Similarly, when a large friction exists between the punched portion of the top sheet and the bottom sheet, the deformation of the bottom sheet would become more difficult and a larger force would be required. Because the deformation of the bottom sheet was mainly executed through the rivet skirt and the interaction between the punched portion of the top sheet and the bottom sheet, the rivet skirt would have a larger deformation as well, resulting in a larger interlock distance.

From Figs. 3, 4, and 5 and Table 2, it can be seen that the thinnest bottom remaining material could be between the tips of the rivet legs and the sides of the joint button, or between the rivet/bottom sheet interface or the top/bottom sheet interface and the bottom of the joint button. For the reference joints, the $T_{\min }$ was between the rivet/bottom sheet interface or the top/bottom sheet interface and the bottom of the joint 
Fig. 11 Static lap shear fracture interfaces. a Reference with original sheet surface condition. b P120 sandpaper ground. c Grit blasted
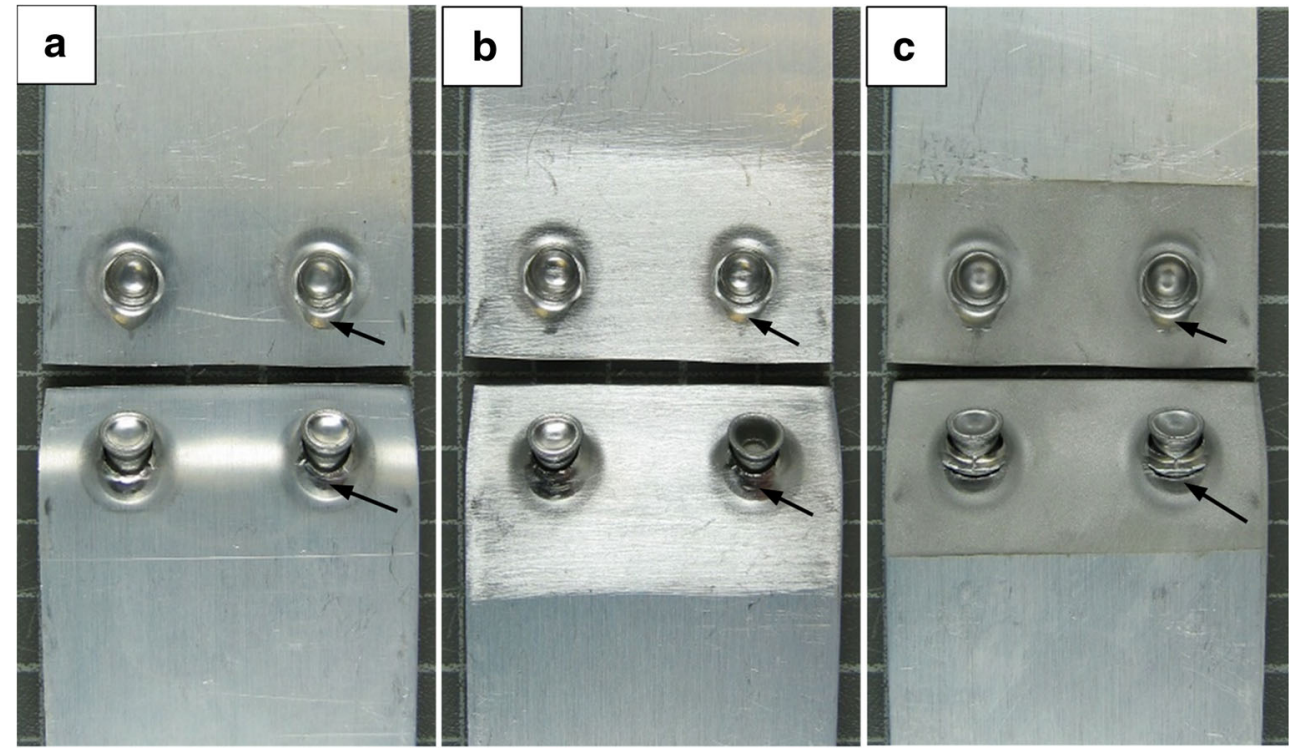

button; however, for other joints with modified sheet surfaces, the $T_{\min }$ was between the rivet legs and the side of the joint button. From Table 2, it can be seen that those surface modifications greatly increased the minimum remaining bottom material thickness to the bottom of the joint button. Washing with hot water and grinding with P120 sandpaper also increased the minimum bottom material thickness to the side of the joint button, but blasting reduced the minimum bottom material thickness to the side of the joint button due to a larger rivet skirt flare.

\subsection{Influence of sheet surface textures/friction on static lap shear strength}

Results from Li et al. [13] pointed out that the friction force between the tip of the punched hole in the top sheet and the edge of the partially pierced hole in the bottom sheet was very important for static lap shear strength. Their results showed that fretting during fatigue increased friction force between the tip of the punched hole in the top sheet and the edge of the partially pierced hole in the bottom sheet, and as a result, the remaining static lap shear strength of the specimens was increased.

Figure 11 shows the lap shear fracture interfaces of the specimens with different surface textures. The arrows indicate the areas where materials slid against each other during lap shear tests. It can be seen that the fracture interfaces for all specimens are similar, where rivets were pulled out from the bottom locking sheet and there were friction marks between the tip of the punched hole in the top sheet and the edge of the partially pierced hole in the bottom sheet. However, for the joints with blasted surface, the top sheet was also ruptured underneath the rivet head. This indicated that the top sheet of the joints with blasted surface sustained a larger force during the lap shear test. For these joints, the interlock distance was much larger than that of the reference joint, as shown in Table 2, which would produce a larger interlocking strength. As a result, to pull the rivet out of the bottom sheet, the top sheet had to sustain a larger force.

In this study, from Fig. 6 and Tables 3 and 4, it can be seen that because of the different sheet surface conditions, such as roughness, amount of remaining lubricant, and residues of hard particles (from grit blasting), the friction severity between the sheet interfaces was different, grit blasted $>$ P120 sandpaper ground $>$ hot tap water washed $>$ reference. It is believed that there were two main factors that were influencing the lap shear strength of the joints studied, i.e. the rivet interlock distance and the friction between the top and the bottom sheet. Because the joints with substrates washed in hot water or ground with sandpaper had similar joint features, especially interlock distance, as the reference joints, as shown in Table 2, the small increase of the lap shear strength $(0.1 \mathrm{kN}$ for hot water washing and $0.3 \mathrm{kN}$ for sandpaper grinding) was mainly caused by the increase of the local friction between the top and the bottom sheet during the lap shear tests. For joints with sheet materials modified by grit blasting, the increase of lap shear strength was much larger $(0.9 \mathrm{kN})$. This larger strength increase was caused not only by the larger friction force between the top and the bottom sheets but also by the larger interlock distances, because for these joints, the joint interlock distance was larger compared with that for the reference joints.

\section{Conclusions}

To study the influence of surface textures on rivet inserting process, joint features and static lap shear strength, one or both of the mating surfaces of the (2+2)AA5754 stack - the bottom 
surface of the top sheet and the top surface of the bottom sheet-were modified by various methods. The following conclusions can be drawn:

1) Hot water washing and sandpaper grinding on AA5754 did not have significant influence on the rivet inserting process and joint features. However, grit blasting on AA5754 increased the rivet setting forces at the beginning and a middle section of the rivet setting displacementforce curve and changed the joint features, with increased interlocks and reduced minimum remaining bottom material thickness $\left(T_{\min }\right)$. It is believed that the difference in the SPR rivet inserting process for the sheet material with different surface conditions was mainly caused by the friction difference between the punched portion of the top sheet and the bottom sheet.

2) The method used to measure the friction between original and modified AA5754 surfaces could not provide consistent results. Stabilized dynamic friction could not be sustained, and the tests normally ended up with wearing between the surfaces. However, the friction tests could give indication of the degree of friction. In this study, the degree of friction for the surfaces measured was in the following order (from high to low): grit-blasted surfaces, P120 sandpaper-ground surface, hot-tap waterwashed surface and original lubricated surface.

3) The lap shear tests showed that hot tap water washing can slightly increase the lap shear strength, P120 sandpaper grinding increased the static lap shear strength further and grit blasting increased the static lap shear strength the most. It can be seen that surface modifications can be used to change the lap shear strength of SPR joints.

Acknowledgements The author would like to thank Innovate UK for the support of this research. The author would also like to thank Henrob Ltd. for providing the rivets for this research, and thank Iain Masters for helping with the friction tests.

Open Access This article is distributed under the terms of the Creative Commons Attribution 4.0 International License (http:// creativecommons.org/licenses/by/4.0/), which permits unrestricted use, distribution, and reproduction in any medium, provided you give appropriate credit to the original author(s) and the source, provide a link to the Creative Commons license, and indicate if changes were made.

\section{References}

1. Krause A, Chernenkoff R (1995) A comparative study of the fatigue behavior of spot welded and mechanically fastened aluminum joints. SAE Technical Paper 950710. doi:10.4271/950710

2. Booth G, Olivier C, Westgate S, Liebrecht F et al (2000) Self-piercing riveted joints and resistance spot welded joints in steel and aluminium. SAE Technical Paper 2000-01-2681. doi:10.4271/ 2000-01-2681

3. Sun X, Stephens EV, Khaleel MA (2007) Fatigue behaviors of selfpiercing rivets joining similar and dissimilar sheet metals. Int $\mathrm{J}$ Fatigue 29(2):370-386

4. Li D, Chrysanthou A, Patel I et al (2017) Self-piercing riveting - a review. Int J Adv Manuf Technol. doi:10.1007/s00170-017-0156-x

5. Abe Y, Kato T, Mori K (2006) Joinability of aluminium alloy and mild steel sheets by self piercing rivet. J Mater Process Technol 177(1-3):417-421

6. Li D, Han L, Thornton M, Shergold M (2010) An evaluation of quality and performance of self-piercing riveted high strength aluminium alloy AA6008 for automotive applications. SAE Technical Paper 2010-01-0223. doi:10.4271/2010-01-0223

7. Hoang NH et al (2013) Failure of aluminium self-piercing rivets: an experimental and numerical study. Mater Des 49(0):323-335

8. Hoang NH et al (2010) Self-piercing riveting connections using aluminium rivets. Int J Solids Struct 47(3-4):427-439

9. Esfahani $\mathrm{M}$ et al (2012) Effect of joining process on the coatings of self-piercing rivets. Adv Mater Res 488-489:1501-1505

10. Han L, Chrysanthou A, Young KW (2007) Mechanical behaviour of self-piercing riveted multi-layer joints under different specimen configurations. Mater Des 28(7):2024-2033

11. Porcaro $\mathrm{R}$ et al (2006) The behaviour of a self-piercing riveted connection under quasi-static loading conditions. Int J Solids Struct 43(17):5110-5131

12. Li D et al (2012) Influence of rivet to sheet edge distance on fatigue strength of self-piercing riveted aluminium joints. Mater Sci Eng A 558(0):242-252

13. Li D et al (2014) The influence of fatigue on the stiffness and remaining static strength of self-piercing riveted aluminium joints. Mater Des 54(0):301-314

14. Li D et al (2013) Influence of rivet tip geometry on the joint quality and mechanical strengths of self-piercing riveted aluminium joints. Mater Sci Forum 765:746-750

15. Li D et al (2012) Influence of edge distance on quality and static behaviour of self-piercing riveted aluminium joints. Mater Des 34: 22-31

16. Han L, Chrysanthou A (2008) Evaluation of quality and behaviour of self-piercing riveted aluminium to high strength low alloy sheets with different surface coatings. Mater Des 29(2):458-468

17. Han L, Young K, Hewitt R, Alkahari M et al (2006) Effect of sheet material coatings on quality and strength of self-piercing riveted joints. SAE Technical Paper 2006-01-0775. doi:10.4271/2006-01-0775

18. Han L, Chrysanthou A, O'Sullivan JM (2006) Fretting behaviour of self-piercing riveted aluminium alloy joints under different interfacial conditions. Mater Des 27(3):200-208

19. Maruyama T, Akagi K, Kobayashi T (2006) Effects of blasting parameters on removability of residual grit. J Therm Spray Technol 15(4):817-821 